\title{
Radar Fusion for Multipath Mitigation in Indoor Environments
}

\author{
Evert I. Pocoma Copa*, Kheireddine Aziz ${ }^{\dagger}$, Maxim Rykunov ${ }^{\dagger}$, Eddy De Greef ${ }^{\dagger}$, André Bourdoux ${ }^{\dagger}$, François Horlin* \\ *Université Libre de Bruxelles (ULB). Bruxelles, Belgium \\ ${ }^{\dagger}$ Interuniversity Microelectronics Centre (imec). Leuven, Belgium \\ \{epocomac,fhorlin\}@ulb.ac.be, \{Kheireddine.Aziz, Maxim.Rykunov, Eddy.DeGreef, Andre.Bourdoux\}@imec.be
}

\begin{abstract}
One of the main challenges of radar-based localization applications in indoor environments is the presence of strong multipath. When the radar bandwidth is large enough, multipath components can be resolved in range but they result in unwanted ghost targets. We propose a novel multipath mitigation approach that exploits the fact that multipaths are highly dependent on the scene geometry. The multipath mitigation approach discards the ghost targets based on the fused information of multiple radars located at different positions in the scene. For such radar fusion, the output of the radar signal processing chain is translated into the world coordinate system that is common for all the radars. We propose a radar alignment approach to estimate the translation and rotation parameters from radar to world coordinate system and vice versa. Our multipath mitigation method is combined with an unscented Kalman filter to improve the localization accuracy. We demonstrate the effectiveness of our complete approach with a real experiment using two radars to detect and track a target in a room with severe multipath.
\end{abstract}

\section{Introduction}

Localization of targets in an indoor environment is of great importance for several applications like surveillance, activity classification, localization of patients in hospitals, crowd monitoring, etc [1]-[3]. Radar sensors measure the kinematics of a target in a direct way, hence they are more accurate than visual sensors like camera. In addition, radars can work in difficult circumstances like bad illumination [4]. Accurate localization and further tracking of targets can be challenging in an indoor environment with all its constraints in area, obstructions, field of view, etc. Additionally, the main drawback when using radars in indoor environments is the presence of strong multipath. It is not possible to distinguish easily the Line-of-Sight (LOS) ray from the multipaths when the radar bandwidth is not large enough. Fortunately, affordable millimeter-wave radars (usually Frequency Modulated Continuous Wave (FMCW) radars) with several $\mathrm{GHz}$ of bandwidth are now available, enabling good range resolution.

Multiple approaches to mitigate the effect of multipaths have been studied in the literature. Such approaches require high complexity operations to remove/mitigate multipaths and most of them are focused on the Range-Doppler-Map (RDM) or the baseband signal. In [5], a signal model of first and second order multipaths is used. The signal model parameters are estimated through a minimization procedure dependent on the samples of the RDM. Then, the multipath effect is removed by means of image deconvolution. In [6], the multipath effect is removed only in the range domain by extracting features from the received energy spectral density. The features are fed to a Neural Network to correct the beat frequency. MIMO Space-Time Adaptive Processing (STAP) techniques are considered in [7]. Finally, Back Projection (BP) algorithm is used in [8], [9] that exploit the fact that multipaths are highly dependent on the scene geometry.

In this paper, we propose a multipath mitigation scheme that exploits the same principle as in [8], [9] . Multiple radars located at different positions receive multipaths coming from different perspectives; therefore, they are independent from one radar to another. The multipath mitigation approach eliminates the detected ghost targets based on the combined information of two radars after the Radar Signal Processing (RSP). Such combination is done in a common coordinate system, and therefore the radar alignment is very important. Hence, our second contribution is a radar alignment approach that estimate the translation and rotation parameters from radar to world coordinate system and vice versa. The last contribution of this paper is an experimental validation of the proposed multipath mitigation approach using two FMCW radars to detect and track targets in a room with severe multipath.

The paper is organized as follows: In section II, we quickly describe the system model, the radar signal processing chain, and the effect of multipath. In sections III and IV, we propose a multipath mitigation approach based on multiple radars and a way to perform a spatial alignment on a common plane of detections provided by both radars. Finally, a proof of concept illustrating the use of the proposed mitigation approach is described in section $\mathrm{V}$.

\section{System Model}

We consider a FMCW radar transmitting a frame of Mconsecutive chirps. The transmitted chirps interact with the objects in the scene. As a result, the received signal is a modified version of the transmitted signal in phase and amplitude. The received signal is then mixed with a replica of the transmitted signal. After discarding terms that are negligibly small in indoor scenarios like the residual phase noise from the transmitter, the beat signal after I/Q sampling can be expressed for the $n^{\text {th }}$ Analog to Digital Converter (ADC) sample and $m^{\text {th }}$ chirp as in (1) as done in [10]. 


$$
y[n, m]=A_{R} e^{j\left(2 \pi f_{b} n T_{f}+\frac{4 \pi}{\lambda} R\left(n T_{f}+m T_{s}\right)\right)}
$$

The parameters in (1) are defined as:

- $A_{R}$ : Received signal power

- $T_{f}$ : Fast-time-axis ADC sampling interval

- $T_{s}$ : Slow-time-axis sampling interval

- $f_{b}=\frac{2 B R(t)}{c T_{c}}$ : Beat frequency

- B: Chirp bandwidth

- $R(t)$ : Target-receiver distance at time $t$

- $T_{c}$ : Chirp duration

- $\lambda$ : Wavelength

Furthermore, in a system with multiple receivers located in a straight line (uniform linear antenna array), an additional phase shift occurs due to the antenna spacing, and thus the beat signal can be expressed as in (2) where $k$ is the antenna index, $d$ is the antenna spacing and $\theta$ is the angle of arrival.

$$
y[n, m, k]=A_{R} e^{j\left(2 \pi f_{b} n T_{f}+\frac{4 \pi}{\lambda} R\left(n T_{f}+m T_{s}\right)+\frac{2 \pi}{\lambda} k d \sin (\theta)\right)}
$$

If multiple targets are present within the radar field-of-view, then the beat signal is the sum of $L$ detected targets located at $\left(R_{l}, \theta_{l}\right)$, as shown in (3).

$y[n, m, k]=\sum_{l=1}^{L} A_{R_{l}} e^{j\left(2 \pi f_{b l} n T_{f}+\frac{4 \pi}{\lambda} R_{l}\left(n T_{f}+m T_{s}\right)+\frac{2 \pi}{\lambda} k d \sin \left(\theta_{l}\right)\right)}$

\section{A. Radar Signal Processing}

The characteristics of detected targets are computed using the RSP shown as a block diagram in Fig. 1. The first step produces the RDM. As can be seen in (1), the fast and slow-time-axes are disentangled, therefore the RDM can be computed by performing first a N-point Fast Fourier Transform (FFT) across the fast-time-axis and then, a M-point FFT across the slow-time-axis. Hence, the RDM is a 2-D map in which the vertical and horizontal axes represent the range and Doppler axes respectively. The RDM must be computed separately for the $K$ receive antennas. The presence of a target is given by the amplitude of the cell for a particular range-Doppler index values.

The second step in the RSP is the Constant False Alarm Rate (CFAR) processing [11]. It selects a cloud of cells where the presence of a target is considered true. The cell selection is based on a comparison between the amplitude of the cell under test and a threshold computed based on the amplitudes of the neighboring cells and a probability of false alarm. The cloud of detected cells are clustered using the Density-Based Spatial Clustering of Applications with Noise (DBSCAN) algorithm [12]. Then, each cluster is represented by the cell of maximum amplitude inside the cluster. The set of coordinates of such representative cells is denoted as $X$. The CFAR and DBSCAN processing are performed on one RDM for detection purposes.

According to (2), it is possible to extract the angle-of-arrival information from the RDM's of several antennas. For that

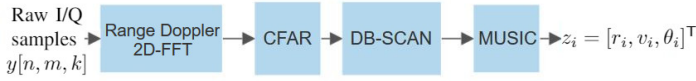

Fig. 1: FMCW Radar signal processing chain

reason, we used a MUSIC algorithm to compute the azimuth angle for each element in $X$, using as input the samples of all the RDM's for the same range-Doppler index.

Finally, a set of detected targets is obtained as a result of the RSP. Each detected target is characterized by range $r$, Doppler velocity $v$, and angle of arrival $\theta$ as shown in (4).

$$
Z=\left\{z_{i}=\left[r_{i}, v_{i}, \theta_{i}\right]^{T} \mid z_{i} \in X\right\}
$$

\section{B. Multipaths and Ghost Targets}

Due to the nature of indoor environments, multiple replicas of the transmitted signal arrive at the receiver. Such signal replicas are denoted as multipaths and they are caused by reflections of the transmitted signal on the interactive objects present in the scene such as walls, people, etc.

Multipaths are highly dependent on the scene geometry and they become weaker as the number of reflections increases. Additionally, they can be classified according to the order of interaction. Only the first and second order multipaths are considered in this work (See Fig. 2). The first order multipaths are interactions in the following sequence: transmittertarget-wall-receiver or transmitter-wall-target-receiver, meanwhile the second order multipaths are interactions in the following sequence: transmitter-wall-target-wall-receiver.

From the receiver perspective, multipaths are observed as incorrectly-assumed valid targets. Therefore, among the $L$ detected targets from the received signal in (3), only a subset of them are valid real targets and the rest are copies of the real targets. These false detected targets are due to multipaths and are denoted as ghost targets. Hence, the set of detected targets $Z$ is the aggregation of the subset of real targets $Z_{d}$ and the subset of ghost targets $Z_{g}$, as expressed in (5).

$$
Z=Z_{d} \cup Z_{g}
$$

\section{Multipath Mitigation}

As explained in section II-B, multipaths are translated into multiple detections of the same target at a different range,

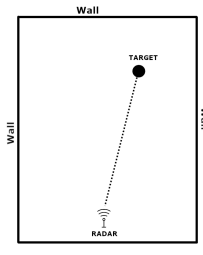

(a)

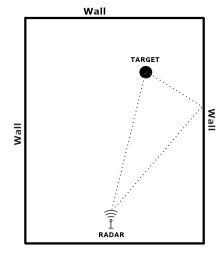

(b)

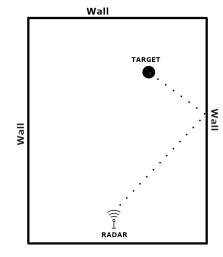

(c)
Fig. 2: Multipath scenarios: a) LOS Ray, b) First order multipath, c) Second order multipath 


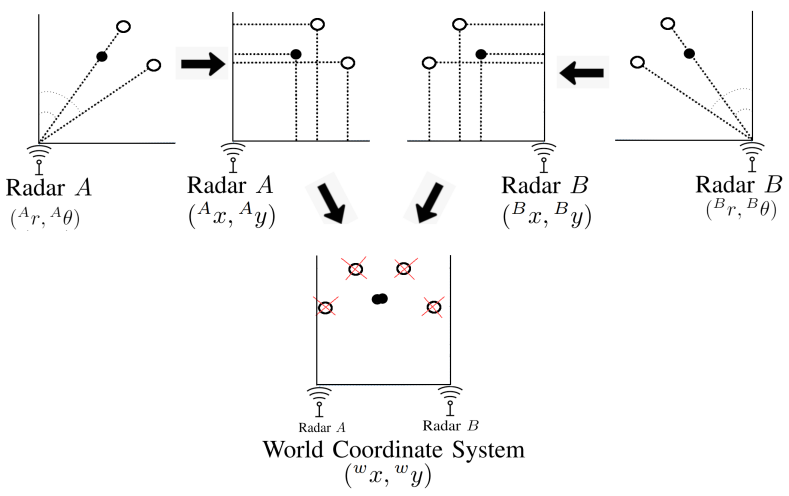

Fig. 3: Multipath mitigation approach: • real target, $\circ$ ghost target

Doppler velocity, and/or angle of arrival. In this paper, only the differences in range and angle are exploited.

Notice that each detected target, expressed as in (4), is measured and expressed in the radar coordinates system. Furthermore, each radar is associated to a different coordinate system with the origin located on the radar itself. Therefore, a common coordinate system of arbitrary origin is chosen denoted as world coordinate system.

The principle of the proposed mitigation approach considers a multi-radar sensor set-up. It is shown in Fig. 3 and it is stated as follows: "Two FMCW radars are located at different positions and both radars illuminate the same scene where $D$ valid targets are present. Expressing the detected targets of both radars in the world coordinate system reveals that the subset of real targets of both radars overlap with each other, whereas the subset of ghost targets do not".

It is important to clearly identify the detecting device and the reference coordinate system for a detected target, therefore the following notation is used: the left superscript represents the coordinate system, the right superscript represents the detecting device. Therefore, the position vector $P_{i}$ of the $i$ - th target can be expressed in multiple ways:

- ${ }^{A} P_{p i}^{A}=\left[{ }^{A} r_{i},{ }^{A} \theta_{i}\right]^{T}$ : Position vector of target $i$ in Polar coordinates of radar A detected by radar $A$.

- ${ }^{B} P_{c j}^{B}=\left[{ }^{B} r_{j},{ }^{B} \theta_{j}\right]^{T}$ : Position vector of target $j$ in Cartesian coordinates of radar B detected by radar $B$.

- ${ }^{w} P_{c i}^{A}=\left[{ }^{w} x_{i},{ }^{w} y_{i}\right]^{T}$ : Position vector of target $i$ in world Cartesian coordinates detected by $\operatorname{radar} A$.

Notice that ${ }^{w} x_{i}$ and ${ }^{w} y_{i}$ are the Cartesian coordinates in the world coordinate system, and ${ }^{A} x_{i},{ }^{A} y_{i}$ are the Cartesian coordinates in the coordinate system of radar $A$. The conversion between Polar and Cartesian coordinates, for target- $i$, is denoted as the function $\Upsilon$ as defined in (6). Notice that, for simplicity, $\theta$ is the complementary angle of the azimuth angle, i.e., $\theta$ is measured related to the radar's $y$-axis.

$$
P_{c i}=\Upsilon\left(P_{p i}\right)=\left[x_{i}, y_{i}\right]^{T} \quad \text { where }: \quad\left\{\begin{array}{l}
x_{i}=r_{i} \sin \theta_{i} \\
y_{i}=r_{i} \cos \theta_{i}
\end{array}\right.
$$

Therefore, the conversion of the coordinates for target $-i$ from the radar Polar coordinate system to the world Cartesian coordinate system is shown in (7), where $R_{A}\left(\phi_{A}\right)$ and $T_{A}$ are the rotation and translation matrices for the radar $A$ defined in (8). Even though (7) and (8) are defined for radar $A$, they are analogous for radar $B$.

$$
\begin{gathered}
{ }^{w} P_{c i}^{A}=R_{A}\left(\phi_{A}\right) \Upsilon\left({ }^{A} P_{p i}^{A}\right)+T_{A} \\
R_{A}\left(\phi_{A}\right)=\left[\begin{array}{ll}
\cos \phi_{A} & \sin \phi_{A} \\
\sin \phi_{A} & \cos \phi_{A}
\end{array}\right] \quad T_{A}=\left[T_{x_{A}}, T_{y_{A}}\right]^{T}
\end{gathered}
$$

The estimation of the Rotation and Translation matrices is addressed in section IV. Hence, they are assumed to be known in this section. Once all the detected targets are expressed in the same coordinate system, only the targets that satisfy the inequality (9) are kept as real targets, i.e., only targets detected by radar $A$ that are close to targets detected by radar $B$ are selected.

$$
\begin{gathered}
\left({ }^{w} P_{c i}^{A}-{ }^{w} P_{c j}^{B}\right) \mathbf{W}\left({ }^{w} P_{c i}^{A}-{ }^{w} P_{c j}^{B}\right)^{T}<d_{\max } \\
d_{\max }=P_{\max } \mathbf{W} P_{\max }^{T}
\end{gathered}
$$

In inequality (9), $d_{\max }$ is the maximum weighted euclidean distance between the detected target $i$ and detected target $j . \mathbf{W}$ is a $2 \times 2$ diagonal weighting matrix with diagonal elements defined as $\operatorname{diag}(\mathbf{W})=\left[w_{x}, w_{y}\right]^{T}$, and $w_{x}$ and $w_{y}$ are the weights on the $x$ and $y$ components respectively. In equation (10) $P_{\max }$ is the vector of maximum difference per component in the world coordinate system.

\section{Radar Alignment}

The process of estimating the rotation and translation parameters $\left[\phi, T_{x}, T_{y}\right]^{T}$ is known as radar alignment. This process is important for the following reason: to use the mitigation approach proposed in section III, the rotation and translation matrices need to be known beforehand. The simplest approach to estimate the parameters of the rotation and translation matrices is to measure them manually. Nevertheless, a small error in the rotation angle $\phi$ will cause a significant error of the Cartesian components for large ranges. Conversely, a small error in the translation parameters $T_{x}, T_{y}$ will cause the same small error in the whole plane.

Therefore, a similar radar alignment approach used in [3] is used in this paper, and it is described as follows:

1) The translation parameters $T_{x}, T_{y}$ are measured manually, i.e. $T^{m}=\left[T_{x}^{m}, T_{y}^{m}\right]^{T}$, the right superindex denotes the manual procedure.

2) The rotation angle $\phi$ is obtained by minimizing the sum of squared alignment errors. Such minimization is shown in (11) for $\operatorname{radar} A$, where $\mathrm{Q}$ is the number of alignment targets, ${ }^{w} P_{c i}^{m}$ is the Cartesian position vector of alignment target $i$ measured manually in the world coordinate system and ${ }^{w} P_{c i}^{A}$ is the Cartesian 
position vector of alignment target $i$ detected by radar $A$ and expressed into the world coordinate system. The term ${ }^{w} P_{c i}^{A}$ is computed as in (7) keeping constant the translation matrix, i.e., $T_{A}=T_{A}^{m}$

$$
\hat{\phi}_{A}=\arg \min _{\phi_{A}} \sum_{i}^{Q}\left\|{ }^{w} P_{c i}^{m}-{ }^{w} P_{c i}^{A}\right\|^{2}
$$

Even though the extraction of the positions of the detected targets by the radars seems to be straightforward, it is not. Due to the static clutter (floor, walls, etc), a static alignment target cannot be detected easily by the radar. Therefore, a nonstatic alignment target that moves in a well known trajectory is used. One of the points of such trajectory is chosen as the alignment target point ${ }^{w} P_{c i}$ needed in (11). A pendulum was chosen as non-static target in the experiments, and the alignment target point is the lowest point of the trajectory, also known as equilibrium point or maximum velocity point.

\section{Tracking}

To show the applicability of the multipath mitigation approach in current real applications, an indoor-people-tracking algorithm has been implemented. Since the movement of people is quite irregular and so, highly non-linear, the Unscented Kalman Filter (UKF) has been chosen as described in the following sections.

\section{A. Tracking Architecture}

The centralized algorithm takes as inputs the detections from both radars after multipath mitigation. Hence a unique track is generated by the UKF as shown in Fig. 4. Furthermore, the UKF receives less erroneous data since the ghost targets are removed before the tracking stage.

\section{B. $U K F$}

To implement the UKF, it is enough to define the process and measurement functions as in [13]. The tracking is done in the world coordinate system where the target position and velocity are tracked in Cartesian coordinates. It is assumed that the axes are independent from each other, and therefore, a Newtonian model can be applied per axis as in [3]. Consequently the state vector $\mathbf{x}$ is defined as in (12), where $x$ and $y$ are the target position and $\dot{x}$ and $\dot{y}$ are the target velocity in the world Cartesian coordinates.

$$
\mathbf{x}=[x, \dot{x}, y, \dot{y}]^{T}
$$

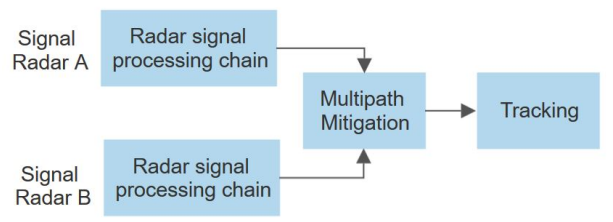

Fig. 4: Radar tracking architecture
1) Process Function: The model chosen in this paper is the constant velocity model detailed in (13), where $\mathbf{x}[k]$ is the state vector at iteration $k, F_{x}$ and $F_{y}$ are the transition matrices for the $x$ and $y$ axes respectively. $F_{x}$ and $F_{y}$ are defined in (14) where $\Delta T$ is the time between iteration $k$ and $k-1$, i.e., the time between consecutive RDM's.

$$
\begin{gathered}
\mathbf{x}[k]=\left[\begin{array}{cc}
\mathbf{F}_{x} & 0 \\
0 & \mathbf{F}_{y}
\end{array}\right] \mathbf{x}[k-1] \\
\mathbf{F}_{x}=\mathbf{F}_{y}=\left[\begin{array}{cc}
1 & \Delta T \\
0 & 1
\end{array}\right]
\end{gathered}
$$

2) Measurement Function: It projects the state space into the measurement space which is defined by the nature of the sensor. In this paper, the measurement space is defined by the radar detections, i.e., by $z=[r, v, \theta]^{T}$. The measurement function is easily defined as shown in the set of equations (15) considering the simplest case scenario i.e., the state and the measurement spaces have the same origin, and only one radar is used. The first two equations of (15) are nothing else than a Cartesian to Polar coordinates conversion. Notice that, for convenience, $\theta$ is defined as the complementary of the azimuth angle. The third equation is a projection of the velocity over the radial axis where $\langle\cdot, \cdot\rangle$ represents the inner product.

$$
z=h(\mathbf{x})=\left\{\begin{aligned}
r & =\sqrt{x^{2}+y^{2}} \\
\theta & =\arctan \left(\frac{x}{y}\right) \\
v & =\frac{1}{r}\left\langle[\dot{x}, \dot{y}]^{T},[x, y]^{T}\right\rangle
\end{aligned}\right.
$$

When a second radar is added to the measurement function, not only the dimensionality increases as shown in (16), but also a change of coordinate system must be considered.

$$
z=\left[z_{A}^{T}, z_{B}^{T}\right]^{T}=\left[r_{A}, v_{A}, \theta_{A}, r_{B}, v_{B}, \theta_{B}\right]^{T}
$$

Since the tracking is done in the world coordinate system, the state space should be first translated to the radar coordinate system. Such translation is given by (17), where the state vector is divided into a position vector $\mathbf{x}_{p}=[x, y]^{T}$ and a velocity vector $\mathbf{x}_{v}=[\dot{x}, \dot{y}]^{T}$. Notice that $R_{A}\left(\phi_{A}\right)$ and $T_{A}$ are the translation matrices computed in section IV, and that the left superscript represents the reference coordinate system.

$$
\left\{\begin{array}{l}
{ }^{A} \mathbf{x}_{p}=R_{A}\left(\phi_{A}\right)^{-1}\left({ }^{w} \mathbf{x}_{p}-T_{A}\right) \\
{ }^{A} \mathbf{x}_{v}=R_{A}\left(\phi_{A}\right)^{-1}\left({ }^{w} \mathbf{x}_{v}\right)
\end{array}\right.
$$

Finally, equation (15) can be used after translation to the radar coordinate system as shown in (18).

$$
z_{A}=h\left({ }^{A} \mathbf{x}\right) \quad ; \quad z_{B}=h\left({ }^{B} \mathbf{x}\right)
$$

\section{Experiments and Results}

We conducted indoor measurements, by placing two FMCW radars on tripods as illustrated in Fig. 5a. We collected a dataset for pedestrians and the recorded dataset has been used to evaluate the different modules of radars alignment and multipath mitigation. Two TI FMCW radars operating 


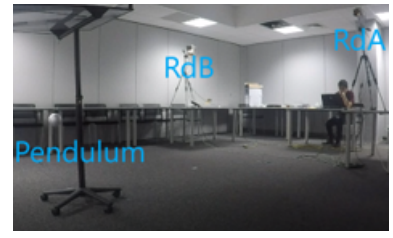

(a)

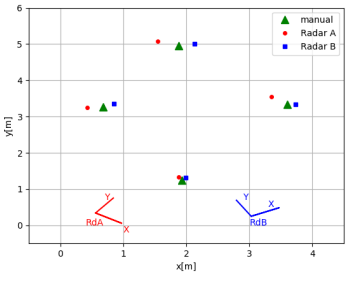

(b)

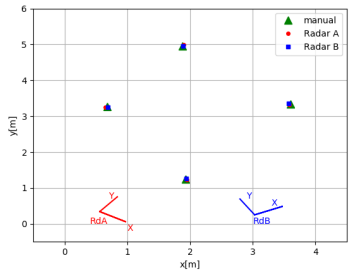

(c)
Fig. 5: Radar alignment: a) Experimental setup, b) Before, c) After.

\begin{tabular}{|l|l|l|l|l|l|l|}
\hline Radar & $T_{x}^{m}[m]$ & $T_{y}^{m}[m]$ & $\phi^{m}\left[{ }^{\circ}\right]$ & $\hat{\phi}\left[^{\circ}\right]$ & $\operatorname{Er}\left(\phi^{m}\right)\left[m^{2}\right]$ & $\operatorname{Er}(\hat{\phi})\left[m^{2}\right]$ \\
\hline A & 0.56 & 0.30 & -30 & -34.33 & 0.31 & 0.0041 \\
B & 3.03 & 0.25 & 25 & 27.09 & 0.13 & 0.0018 \\
\hline
\end{tabular}

TABLE I: Alignment parameters

in MIMO mode are used in our experiments. Both radars were configured with non-overlapping frequencies to enable simultaneous operation. Radar $A$ works from $77 \mathrm{GHz}$ to $79 \mathrm{GHz}$ and radar $B$ works from $79 \mathrm{GHz}$ to $81 \mathrm{GHz}$. This also means that the range resolution is $7.5 \mathrm{~cm}$. Considering the fact that the both radars operate at different acquisition rates, we time-stamped the measurements and updated the system with measurements having very close time-stamps. This allows obtaining a uniform synchronization.

\section{A. Radar Alignment}

In our experiment, four alignment target points were considered at the following locations given in meters: ${ }^{w} P_{c 1}^{m}=$ $[0.68,3.26]^{T},{ }^{w} P_{c 2}^{m}=[3.60,3.34]^{T},{ }^{w} P_{c 3}^{m}=[1.93,1.25]^{T}$, and ${ }^{w} P_{c 4}^{m}=[1.88,4.96]^{T}$. A pendulum was oscillating at each of these locations, and only the maximum-velocity point or point of equilibrium was taken as explained in section IV. The height of the radars in the 2D case is irrelevant, nonetheless it is worth to mention that the radars are placed at the same height. In Fig. 5b and 5c, The manually measured and radar detected alignment points are plotted in the world coordinate system. It is observed that for small errors in azimuth, the alignment error is larger for large ranges. Finally, Fig. 5c shows the result after the radar alignment with the method proposed in this paper. The manually measured and final estimated alignment parameters of both radars are displayed in Table I, where the right super-index $m$ is for manually measured parameters. $\operatorname{Er}\left(\phi^{m}\right)$ is the squared alignment error using the manually measured azimuth angle, and $\operatorname{Er}(\hat{\phi})$ is the squared alignment error using the estimated azimuth angle. Notice that once the radar alignment procedure is completed, the alignment targets are removed from the scene.
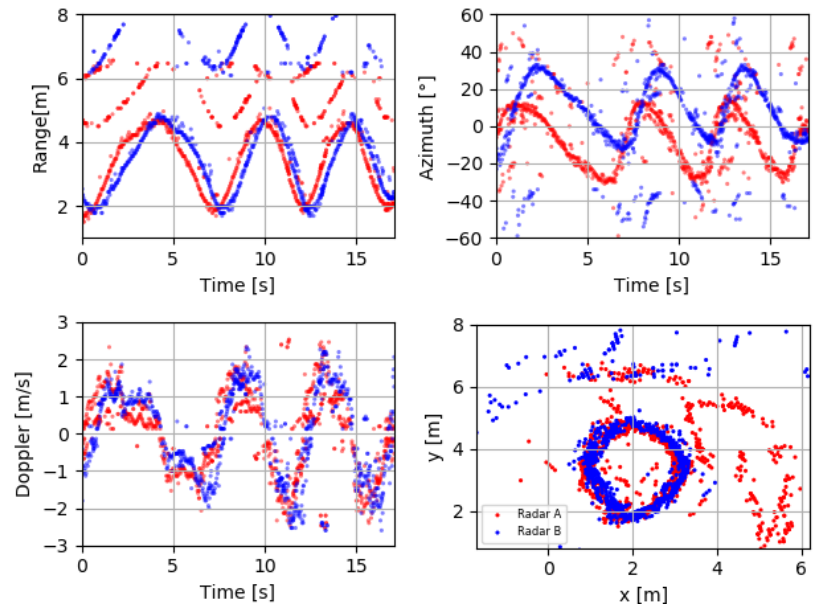

Fig. 6: Radar measurements before multipath mitigation
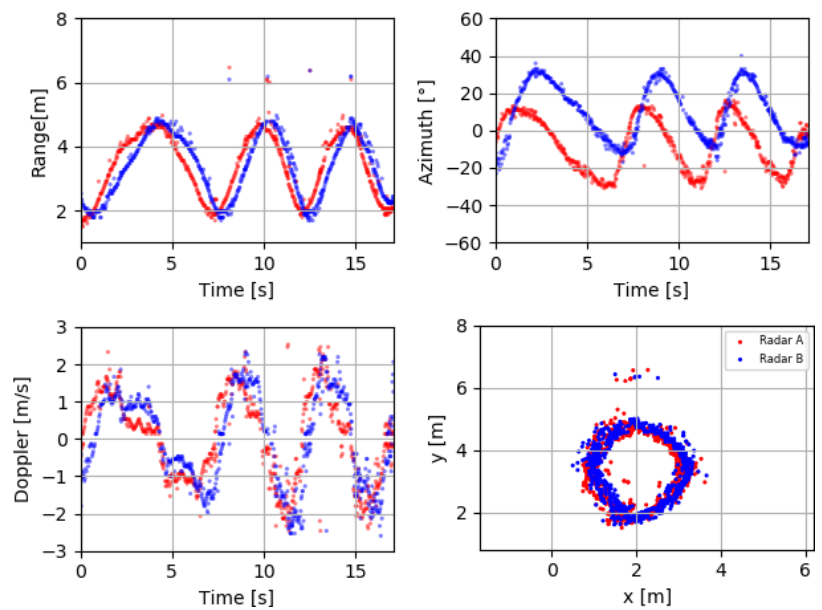

Fig. 7: Radar measurements after multipath mitigation

\section{B. Multipath Mitigation}

One person was moving from one alignment target position to another in the following sequence: $P_{c 1}, P_{c 2}, P_{c 3}, P_{c 4}$. The radar measurements are displayed in Fig. 6 where the ghost targets caused by multipaths are easily observed. For instance, in the range plot of the same figure, the real targets are the red and blue lower curves, meanwhile the ghost targets appear at a longer range with less intensity.

The proposed multipath mitigation approach stated in section III was used with the following parameters: since axes $x$ and $y$ are independent and equally relevant for the tracking, matrix $(\mathbf{W})$ has the following diagonal elements $\operatorname{diag}(\mathbf{W})=[1,1]^{T}$. The average shoulder width of a person is $40 \mathrm{~cm}$, and thus it was taken as the maximum distance per component, i.e. $P_{\max }=[0.4,0.4]^{T}$. As a result the ghost targets where removed as can be seen clearly in Fig. 7.

The Multipath mitigation approach proposed in section III is a simple and easy to implement algorithm. Nonetheless, it is not perfect and needs an additional improvement step, as discussed hereafter: 

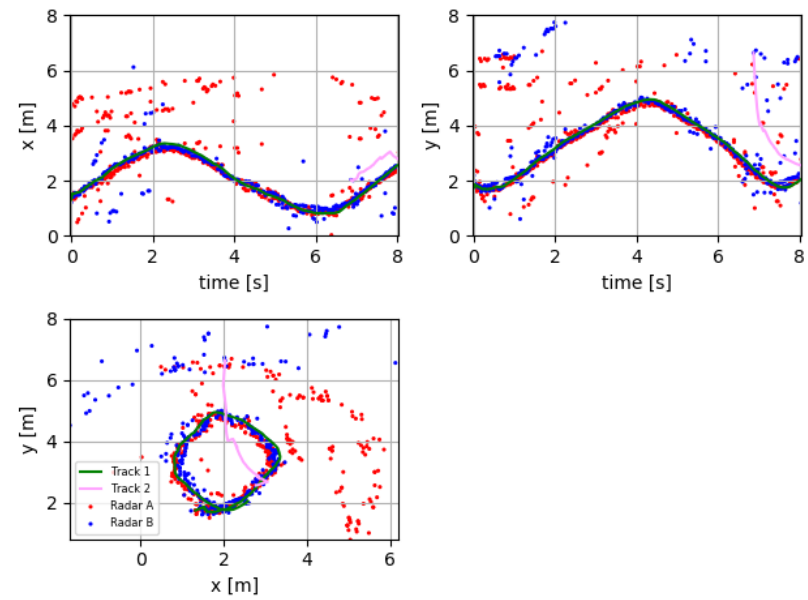

Fig. 8: Indoor people tracking result before multipath mitigation

- Remaining ghost targets: In some cases, ghost targets and real targets are close from each other, e.g. when the person walks next to the side walls. Therefore, the mitigation approach fails to discriminate real targets from ghost (Fig. 7).

- Occlusion limitation: The mitigation approach works as long as both radars detect the real target. In the case where only one of them detects it, the valid real detection is discarded as if it were a ghost target detection.

\section{Tracking}

If no multipath mitigation is performed, ghost targets can lead to generation of erroneous tracks. Such tracks can be eliminated by the track management; however, the required time to eliminate the track can be long. An example of erroneous track is shown in Fig. 8, where the tracking is performed in the world coordinate system.

Thanks to the mitigation approach proposed in Section III, the ghost targets are removed before the tracking. Hence, it not only reduces the computational load of the UKF but also prevents the creation of erroneous tracks as shown in Fig. 9.

\section{Conclusion}

An efficient and easy to implement multipath mitigation approach was proposed. This approach exploits an arrangement of multiple radars located at different locations, taking advantage of the different measurements of the same scene. We developed a complete suite of algorithms to calibrate the radars in the scene and to fuse the information provided by the radars to get multipath-free detections. Furthermore, we demonstrated the feasibility and efficacy of the method in a people tracking application in a multipath environment.

\section{References}

[1] G. Santhiya, K. Sankaragomathi, S. Selvarani, and A. N. Kumar, "Abnormal crowd tracking and motion analysis," in 2014 IEEE International Conference on Advanced Communications, Control and Computing Technologies, 2014, pp. 1300-1304.
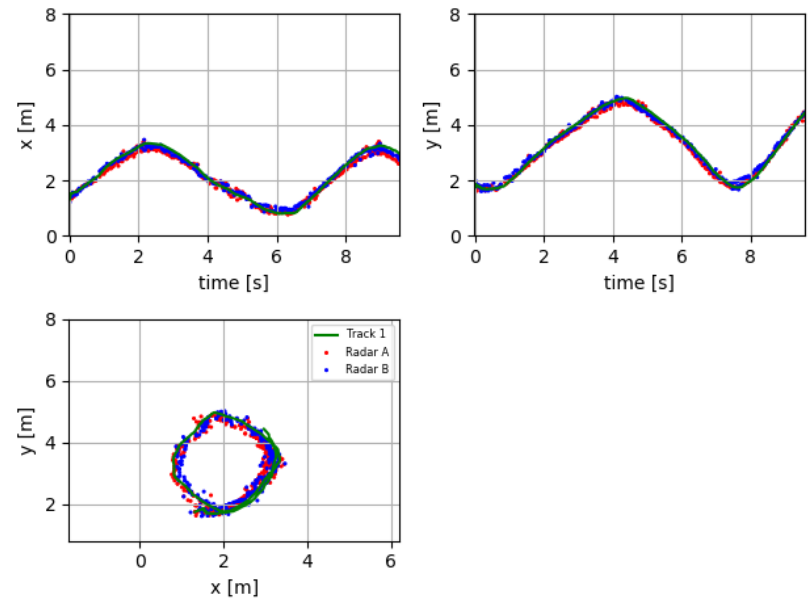

Fig. 9: Indoor people tracking result after multipath mitigation

[2] W. C. Lee, F. H. Hung, K. F. Tsang, C. K. Wu, and H. R. Chi, "Rss-based localization algorithm for indoor patient tracking," in 2016 IEEE 14th International Conference on Industrial Informatics, 2016, pp. 10601064.

[3] F. Botha, "Data fusion of radar and stereo vision for detection and tracking of moving objects," Master's thesis, Stellenbosch University, 2017. [Online]. Available: https://bit.ly/38VhpTy

[4] J. Steinbaeck, C. Steger, G. Holweg, and N. Druml, "Next generation radar sensors in automotive sensor fusion systems," in 2017 Sensor Data Fusion: Trends, Solutions, Applications (SDF), 2017, pp. 1-6.

[5] B. D. Rigling, "Urban rf multipath mitigation," IET Radar, Sonar Navigation, vol. 2, no. 6, pp. 419-425, 2008.

[6] B. Waldmann, A. Goetz, and R. Weigel, "An ultra wideband positioning system enhanced by a short multipath mitigation technique," in 2009 IEEE MTT-S International Microwave Workshop on Wireless Sensing, Local Positioning, and RFID, 2009, pp. 1-4.

[7] J. Yu and J. Krolik, "Mimo multipath clutter mitigation for gmti automotive radar in urban environments," in IET International Conference on Radar Systems, 2012, pp. 1-5.

[8] S. Guo, G. Cui, L. Kong, and X. Yang, "An imaging dictionary based multipath suppression algorithm for through-wall radar imaging," IEEE Transactions on Aerospace and Electronic Systems, vol. 54, no. 1, pp. $269-283,2018$

[9] S. Guo, X. Yang, G. Cui, Y. Song, and L. Kong, "Multipath ghost suppression for through-the-wall imaging radar via array rotating," IEEE Geoscience and Remote Sensing Letters, vol. 15, no. 6, pp. 868-872, 2018.

[10] A. Ahmad, J. C. Roh, D. Wang, and A. Dubey, "Vital signs monitoring of multiple people using a fmcw millimeter-wave sensor," in 2018 IEEE Radar Conference, 2018, pp. 1450-1455.

[11] A. Jalil, H. Yousaf, and M. I. Baig, "Analysis of cfar techniques," in 2016 13th International Bhurban Conference on Applied Sciences and Technology (IBCAST), 2016, pp. 654-659.

[12] I. Babur, J. Ahmad, B. Ahmad, and M. Habib, "Analysis of dbscan clustering technique on different datasets using weka tool," Science International, vol. 27, pp. 5087-5090, 2015.

[13] E. A. Wan and R. Van Der Merwe, "The unscented kalman filter for nonlinear estimation," in Proceedings of the IEEE 2000 Adaptive Systems for Signal Processing, Communications, and Control Symposium, 2000, pp. 153-158. 